Revista de Psicología Vol. 34 (2), 2016 (ISSN 0254-9247)

\title{
Regulación del estrés y emociones con actividades gráficas y narrativa expresiva
}

\author{
Minerva Vanegas-Farfano ${ }^{1}$, Mónica González Ramírez² y Rodrigo Cantú Guzmán ${ }^{3}$ \\ Universidad Autónoma de Nuevo León, México
}

Se evalúa el impacto de las instrucciones dadas para el uso de actividades gráficas y escritura expresiva como una forma de apoyo en el manejo del estrés y las emociones a partir de seis grupos experimentales con treinta estudiantes universitarios. Los efectos fueron estimados mediante la adaptación mexicana de la Escala de Estrés Percibido y la adaptación de Escala de Afecto Positivo y Negativo. Los grupos fueron similares en su estrés percibido y en emociones en el tiempo 1. Los resultados indican que ambas variables mejoran tras la intervención (tiempo 2), mostrando diferencias entre los grupos. Se concluye que es necesario evaluar el tipo de instrucciones en ambientes experimentales y clínicos considerando estas diferencias.

Palabras clave: diseño experimental; terapia artística; estrés percibido; regulación emocional.

\section{Stress and emotion regulation with graphic activities and expressive narrative}

The present study evaluated instructions in graphic and expressive writing tasks as stress and emotion regulation activity aids. Six experimental groups were composed of thirty university students. Effects were measured with the Mexican versions of the Perceived Stress Scale (PSS) and the Positive and Negative Affect Schedule (PANAS). The groups were similar in

1 Estudiante de doctorado por la Facultad de Psicología de la Universidad Autónoma de Nuevo León, México. Dirección postal: Universidad Autónoma de Nuevo León, UANL, Fac. de Psicología. Av. Universidad S/N Ciudad Universitaria, San Nicolás de los Garza Nuevo León, C.P. 66451. México. Contacto: minerva.vanegas@gmail.com

2 Doctora en Metodología de las Ciencias del Comportamiento. Miembro del Sistema Nacional de Investigadores (Nivel I). Catedrática de la Facultad de Psicología de la Universidad Autónoma de Nuevo León, México. Dirección postal: Universidad Autónoma de Nuevo León, UANL, Facultad de Psicología. Av. Universidad s/n. Ciudad Universitaria, San Nicolás de los Garza. Nuevo León, C.P. 66451, México. Contacto: monygzz77@yahoo.com

3 Doctor en Psicología. Miembro del Sistema Nacional de Investigadores. Psicólogo clínico e investigador de los Servicios Médicos de la Universidad Autónoma de Nuevo León y del Departamento de Bioquímica de la Facultad de Medicina de la Universidad Autónoma de Nuevo León. Dirección postal: Universidad Autónoma de Nuevo León, UANL, Facultad de Psicología. Av. Universidad s/n. Ciudad Universitaria, San Nicolás de los Garza. Nuevo León, C.P. 66451, México. Contacto: rodrigocantu@yahoo.com 
the perceived stress and affect measures in time 1. Results demonstrated positive changes in both variables in time 2,for the intervention groups. The results indicate that it is necessary to evaluate the types of instructions given in experimental and clinical settings.

Key words: Experimental design; art therapy; perceived stress; emotion regulation.

\section{Regulaçáo de estresse e emoçóes com atividades gráficas e narrativa expressiva}

O propósito do estudo foi avaliar o impacto das instruçóes dadas para o uso de atividades gráficas e escritura expressiva como uma forma de apoio no controle do estresse e das emoçóes. A amostra foi formada por trinta estudantes universitários. Deste modo formaram-se seis grupos experimentais, cujos efeitos foram medidos mediante a adaptação mexicana da Escala de Estresse Percebido e a adaptação da Escala de Afeto Positivo e Negativo. Os grupos foram similares no estresse percebido e nas emoçóes no tempo 1. Os resultados indicam que ambas variáveis melhoram depois da intervenção (tempo 2); mas com diferenças entre os grupos. Deste modo se conclui que é necessário avaliar o tipo de instruçóes em ambientes experimentais e clínicos considerando estas diferenças.

Palavras-chave: desenho experimental; terapia artística; estresse percebido; regulação emocional. 
Preservar un estado de salud que permita llevar a cabo lo cotidiano es una ocupación de toda nación y persona, de la que en ocasiones solo se tiene atención cuando se encuentra amenazada. Un fenómeno inscrito en este tipo de dolencias es el estrés psicológico, cuyos efectos se presentan y comentan en prácticamente cualquier esfera de la vida e incluso como problema de salud pública en diversas áreas del mundo (American Psychological Association [APA], 2010; González et al., 2012).

El estrés psicológico se redefine como un inconveniente de salud necesario a observar (APA, 2010), tras el reconocimiento de su interferencia en la habilidad que tiene la persona para llevar una vida normal, por sí mismo o asociado a otras dolencias. Relacionado con problemas tanto físicos como psicológicos, el estrés no surge ni persiste en el aislamiento. Como producto de la percepción de situaciones que provocan cambios en los estados emocionales, comportamientos e ideas en la búsqueda de su control o resolución (Casari, Anglada \& Daher, 2014; Eschleman, Alarcon, Lyons, Strokes \& Schneider, 2011) el estrés psicológico se observa dentro de ambientes laborales (Leka, Griffiths \& Cox, 2004), educativos e interpersonales de distintos niveles. Asimismo se sabe que este se relaciona con otros problemas, como los trastornos de ansiedad o afectivos, tanto en niños como adultos.

No todas las personas desarrollan un nivel de estrés que sea reportado como clínico o propenso a desencadenar alguna dolencia. Una de las razones de ellos es quizás la formas como son afrontadas las situaciones estresantes a lo largo de la vida. A fin de hacer frente al estrés cotidiano actualmente existe un amplio repertorio de actividades recomendadas para manejarle como el hacer deporte o caminar, escuchar música, leer, ver la televisión, utilizar videojuegos, es decir, utilizar algún tipo de pasatiempo. La razón que se da para su uso es que estos permiten tanto una mejora general de la salud como efectos sobre las emociones (APA, 2010; Linley et al., 2012). Como un medio capaz 
de fomentar la expresión y con ello, el manejo de conflictos, de los anteriores pasatiempos las actividades como el dibujo o la pintura son utilizadas también en ambientes clínicos con fines terapéuticos (American Art Therapy Association, AATA, 2013).

\section{La actividad artística y su relación con la salud}

El uso de la actividad artística con fines terapéuticos tiene una historia que remonta al menos hacia la era moderna (Kandel, 2012). Su trabajo, avalado por un creciente interés en la investigación, permite considerarle como una forma de intervención en equipos multidisciplinarios o tratamiento único en plazas como hospitales, centros de atención psiquiátrica o psicológica, espacios de rehabilitación, centros para el desarrollo del bienestar, escuelas, centros geriátricos o dentro de la práctica privada (AATA, 2013). Asimismo, frente a otros tipos de terapia se le atribuye el dar acceso a aspectos de la vida psíquica que desde otros enfoques no es posible con las herramientas usuales (Marxen, 2011).

El estudio de la actividad artística se ha concentrado en poblaciones con un diagnóstico que compromete la salud física o psicológica. Recientemente el interés por sus efectos en población no clínica crece y comienza a publicarse bajo el escrutinio de actividades como la escritura, la pintura y la música (Dalebroux, Goldstein \& Winner, 2008). A pesar de la diversificación en su uso, la falta de una fundamentación de su costo-beneficio, conocimiento de contraindicaciones o la identificación de las variables psicológicas que varían bajo las intervenciones terapéuticas o en otras condiciones pareciese limitar tanto su aceptación como desenvolvimiento teórico (Crawford \& Patterson, 2007; Muri, 2007).

\section{El estudio de la actividad gráfica y su relación con el estrés y las emociones}

El desconocimiento sobre las causas o mecanismos subyacentes al uso de actividades artísticas y el efecto reportado por quien la practica 
Regulación del estrés y emociones con actividades gráficas y narrativa expresiva / Vanegas-Farfano et al.

parece, de acuerdo a los reportes otorgados por estudios cualitativos relacionarse con la descarga de las emociones, una acción orientada a la auto regulación emocional (Drake \& Winner, 2012b; Dalebroux, Goldstein \& Winner, 2008). Las actividades gráfico-artísticas promueven el uso de estrategias ante eventos estresantes: permiten su manejo activo al dar la oportunidad para expresarles y proponer respuestas (Huss, 2012); o bien, fungen como estrategia pasiva, dando pie a un espacio de tranquilidad (Reynolds, 2010; Singer, Götze, Buttstädt, Geue, Momenghalibaf \& Böhler, 2010).

Dentro de un ambiente clínico, la elaboración gráfica, acompañada de una narración realizada por el paciente en sincronía con el terapeuta permite desarrollar un modelo similar a lo observado en investigaciones sobre escritura expresiva, en donde tras exponer una situación narrándola en papel, se logra la integración de información emocional sobre las propias experiencias (Piolat \& Bannour, 2011); proceso que se esperaría diese coherencia entre hechos y su narrativa, como sucede en diversas intervenciones psicológicas (Drake $\&$ Winner, 2012a). Asimismo, investigaciones anteriores a la presente han mostrado que en ambientes clínicos, intervenciones que incluyen tanto la narrativa como la expresión artística permiten a los pacientes el reconocimiento de la práctica de un afrontamiento activo ante la enfermedad así como la valoración de los aspectos psicosociales que influyen sus respuestas ante la misma (Cantú \& Álvarez, 2011).

No solo el enfocarse en el problema deriva en su solución o un estado saludable: distraerse es desde la infancia, una de las estrategias más comunes para regular las emociones. De acuerdo con Drake y Winner (2012b) el enfocar la atención en algo distinto al estresor o el espacio vinculado a la agitación emocional permite desde edades tempranas una disminución significativa de los sentimientos negativos. Asimismo y como ya ha sido mencionado anteriormente, el estrés psicológico en adultos es también manejado mediante el uso de actividades lúdicas. Ambas categorías —enfoque o distracción — son por sí mismas mutuamente excluyentes; pero como ha sido seńalado, a un nivel de variable o fenómeno psicológico presenta beneficios a la salud aun cuando se trata 
de realizar un mismo tipo de actividad, por ejemplo, una pintura. Cabe señalar que si bien estas mismas respuestas —enfoque en el problema o su distanciamiento - ya se estudian en función a problemas específicos como es el prepararse para un examen, en situaciones como esta ya se conoce una distinción en su uso con respecto a la edad o nivel de aprendizaje adquirido por los participantes (Casari et al., 2014); no así en el empleo de actividades artísticas.

\section{Respuestas provenientes de la psicología cognitiva}

Desde el desarrollo teórico del estrés y el uso de estrategias de afrontamiento, es ampliamente conocido que el centrarse en un problema puede llevar a su resolución cuando se logra adquirir y aplicar adecuadamente los conocimientos. Contrario a ello, el distraerse de la situación preocupante es considerado como una forma de evitación que puede incluso caer en la disfuncionalidad o lo no adaptativo (Cano, Rodríguez y García, 2007). Desde la psicología cognitiva existe otra forma de vislumbrar estos mismos mecanismos, observándose como una forma de afrontamiento antagónica a su solución. Cognitivamente esta acción es más compleja: distraerse puede guiar los pensamientos o conductas hacia otras incompatibles con la situación estresante (distracción activa), o bien concentrar la atención en materiales considerados como emotivamente neutros o cargados positivamente (distracción pasiva). En otras palabras, puede permitir el manejo del estrés y postergar la solución del problema a un momento más adecuado. Asimismo, en estudios experimentales se ha visto que esta diferenciación tiene incluso un impacto en los estados emocionales derivados del distraerse u orientarse a una respuesta inmediata (Webb, Miles $\&$ Sheeran, 2012).

Tras vislumbrar cómo la actividad expresiva se inserta en la intervención terapéutica con ambos resultados, nacen algunas interrogantes: dadas las implicaciones que tiene el enfocar la atención en un problema y el distraerse de este ¿qué implica dicha actividad? ¿En función a qué se obtienen unos u otros? Y, ¿podría beneficiarse igualmente de la actividad sin apoyo de un terapeuta? Considerando lo anterior, y que la población de estudiantes universitarios es un grupo poco explorado en 
el campo de las actividades artísticas como intervención para la salud, pero reconocido como una parte de la población cuyo estrés es considerado como generalizado (García-Ros, Pérez-Blasco \& Natividad, 2012; Martín, 2007) este estudio enfoca las anteriores interrogantes a búsqueda de las diferencias que aportan las instrucciones presentadas a los participantes frente a una situación estresante al solicitarles enfocarse en el problema o distraerse, activa o pasivamente, mediante el empleo de una actividad gráfica. Para lograrlo se planteó como objetivo comparar cambios en las emociones y el estrés percibido en función al empleo de las instrucciones presentadas para elaborar un dibujo con y sin la elaboración previa de un texto sobre una situación estresante personal actual. Se contempla como hipótesis de trabajo que se modifica el estrés percibido o los estados emocionales mediante una actividad artística.

\section{Método}

Se empleó un diseño experimental factorial mixto de una sola sesión orientado al aislamiento de los efectos de la actividad artística y narrativa del suceso estresante. La comparación de los cambios en las variables dependientes - estrés percibido y emociones - se hizo mediante una comparación pre test y post test como sugieren Kerlinger y Lee (2002). La condición de intervención separó el trabajo gráfico/artístico como distracción activa, distracción pasiva y enfoque en la situación estresante. Por otro lado, la narrativa del suceso se efectuó antes o después de la intervención, generando con ello seis grupos: distracción activa/narración, distracción activa/sin narración; distracción pasiva/narración, distracción pasiva/sin narración; enfoque/narración y enfoque/sin narración.

\section{Participantes}

Participaron treinta estudiantes de una facultad de odontología de la zona metropolitana de Monterrey, México a partir de un muestreo aleatoria que consideró como criterios de inclusión: llevar carga semestral completa, sentirse capacitado para realizar una actividad 
gráfica (dibujo) a nivel no profesional y poder identificar una situación estresante que influya actualmente en su vida. Como criterios de exclusión consideró una o varias de las siguientes situaciones: periodo de duelo, tener confirmado un diagnóstico de ansiedad, depresión o estrés post-traumático y/o de abuso de sustancias.

\section{Medidas}

A continuación, se presentan los instrumentos de evaluación utilizados en este proyecto.

Escala de Estrés Percibido (PSS) de Cohen, Kamarak y Mermelstein, elaborado en 1983 y adaptado en México por González y Landero (2007). Es un inventario autoadministrable que evalúa el grado en que la persona percibe su vida como estresante. Consta de catorce ítems con puntuación Likert de 0 a 4: "Nunca", "Casi nunca", "De vez en cuando", "A menudo" y "Muy a menudo (4). Cuenta con siete ítems inversos $(4,5,6,7,9,10$ y 13). Su evaluación se realiza mediante la sumatoria de todos sus ítems. Presenta un coeficiente de fiabilidad de .83. En el presente estudio su fiabilidad fue de .75 en el primer tiempo y .72 en el segundo tiempo.

Escala de Afecto Positivo y Negativo (PANAS) de Watson y Clark (1988) en su validación y versión corta en castellano para población mexicana realizada por Robles y Páez (2003). Este inventario, también autoadministrable, mide dos dimensiones de emocionalidad (afecto positivo y negativo) y cuenta con diez ítems para cada una de estas. En el presente estudio se utilizó en su valoración de la última semana, que cuenta con una fiabilidad interna de .85 para el afecto positivo y .81 para el negativo en la población mexicana. Durante el primer tiempo su fiabilidad fue de .86 para el afecto positivo y .82 para el afecto negativo en el primer tiempo; mientras que en segundo fue de .96 para el afecto positivo y de .94 para el afecto negativo.

International Affective Picture System (IAPS) de Lang, Bradley y Cuthbert (2008). Es una serie de imágenes cuya normatividad y desarrollo se centra en la generación de estímulos para uso experimental en torno a las emociones y atención. Fueron utilizadas como parte del 
material entregado bajo la condición de distracción pasiva una serie de diez imágenes tomadas al azar considerando para ello que su valencia fuese neutra en los rubros de placer, estimulación y dominancia.

En cuanto al material gráfico se utilizó una hoja tamaño carta de papel fabriano de gramaje medio, doce tizas de colores y un lápiz con borrador.

\section{Procedimiento y procesamiento de la información}

Esta investigación fue realizada en las aulas universitarias con la autorización del profesor responsable del material. La participación fue voluntaria y anónima. El tiempo estimado para efectuar la sesión fue de 45 minutos. Antes de su inicio fue explicado a los alumnos los criterios de inclusión y exclusión para la participación y en qué consistía la investigación, y luego se firmó el consentimiento informado.

Se entregó a los participantes los cuestionarios y material gráfico dentro de un sobre cerrado el cual incluía impreso un formato que especificaba la naturaleza del proyecto, sus objetivos y aspectos éticos. Ya que cada grupo de intervención contaba con instrucciones distintas, los sobres eran identificados con un color diferente. La entrega de los mismos se hizo de forma aleatoria. Una vez que contestaban los datos del primer grupo de inventarios (pre test, tiempo uno) se daba la indicación de realizar la actividad gráfica con el material entregado, enfatizando la acción correspondiente al tipo de intervención o grupo en el cual participaba: «copiar una de las imágenes incluida en el material» en el caso de los participantes de la condición distracción pasiva; "representar el objeto o situación estresante que haya documentado/ pensado en los cuestionarios anteriores» para aquellos que trabajaron la condición de enfoque o "representar un objeto o situación que le permita distraerse de la situación estresante que haya documentado/ pensado en los cuestionarios anteriores» en el caso de los participantes bajo la condición de distracción activa. Estas mismas instrucciones fueron presentadas en el pizarrón de las aulas señalando a qué color pertenecía cada una de ellas. 
En cuanto a la narrativa, se solicitó que en una hoja entregada en blanco escribiesen sobre una situación estresante que estuviesen viviendo. Sobre esta se aclaró que por estresante habría de entenderse una situación que les cause problemas, les haga sentir mal o les cueste mucho enfrentarse Asimismo se dio la libertad de que esta se enfocase al ámbito familiar, escolar, de pareja o amistad y laboral. La ejecución de este texto fue elaborada por todos los grupos, antes de la actividad gráfica para aquellos que participaban en la condición de narrativa y después de esta en la condición sin narrativa. Haber optado por el empleo de situaciones estresantes provenientes de la vida cotidiana de los participantes obedeció a las consideraciones de autores como Montes, Rodríguez y Serrano (2014) quienes la señalan como necesario a tomar en cuenta, la relación actual de la persona con el evento pues estas pueden tener un efecto directo en las emociones vinculadas con los resultados; por lo que una situación ficticia experimental no necesariamente habría de dar los mismos resultados que el trabajo con problemas reales.

En un tercer paso, los estudiantes llenaron el segundo grupo de inventarios (post test, tiempo dos). Por último, se pidió a los alumnos que hicieran una lista o bien un dibujo de «actividades que puedan realizar a fin de obtener resultados positivos a la situación estresante de acuerdo a sus habilidades actuales» considerando la posible presencia de emociones negativas derivadas del manejo de sucesos estresantes y las recomendaciones éticas señaladas en estudios similares (Szczygiel, Buczny \& Bazinka, 2012). Se solicitó la devolución del material de trabajo dentro del mismo paquete. Una vez recolectado fue empleado el paquete estadístico SPSS en su versión 19.0 para Windows en todos los análisis estadísticos.

\section{Resultados}

En cuanto a las variables sociodemográficas de edad, sexo y ocupación de los participantes, sus resultados se presentan en la tabla 1 . 
Como se observa en esta los participantes se encontraban dentro del rango de edad de los 21 a los 24.4 años y pertenecían a los semestres escolares de séptimo a noveno.

\section{Tabla 1}

Características sociodemográficas de los seis grupos experimentales

\begin{tabular}{cccccccc}
\hline & & \multicolumn{7}{c}{ Grupo } \\
& & 1 & 2 & 3 & 4 & 5 & 6 \\
\cline { 2 - 8 } Edad & $M$ & 21.8 & 21.4 & 21 & 21 & 24.4 & 23.2 \\
& $D E$ & 1.6 & 1.14 & 0.7 & 1.22 & 2.3 & 2.58 \\
\hline \multirow{2}{*}{ Sexo $(n)$} & Masculino & 2 & 2 & 1 & 2 & 4 & 1 \\
& Femenino & 3 & 3 & 4 & 3 & 1 & 4 \\
\hline \multirow{2}{*}{ Semestre $(n)$} & 7 & & 5 & 5 & 4 & & 2 \\
& 8 & 5 & & & 1 & 1 & 1 \\
\hline \multirow{2}{*}{$\begin{array}{c}\text { Ocupación } \\
(n)\end{array}$} & 9 & & & & & 4 & 2 \\
\hline
\end{tabular}

La exploración de las situaciones que consideraban como estresantes los participantes sugirió la extracción de seis categorías. Tres principales y tres que corresponden a un modelo mixto de esas, es decir, al reporte de situaciones en donde era claro para el participante que a pesar de estar ubicado el problema en una esfera de su vida este incidía o generaba estresores en otras o bien se sumaban a los ya expuestos en la narración. Las tres categorías principales fueron:

a) Académico. Situaciones propias del contexto escolar como exámenes, entrega de trabajos, participación en proyecto o materias específicas como prácticas académicas. Ejemplo: “...el tener que buscar pacientes para los tratamientos"

b) Laboral. Incluye problemas relacionados con el espacio de trabajo, personas que laboran con ellos (jefes, compañeros de trabajo), las demandas del puesto y la relación tiempo-experiencia en función a estas. Un ejemplo de ella es: "No tengo trabajo ya, por una semana". 
c) Personal. Se incluyeron en esta categoría los problemas relacionados con la familia, amistades y/o pareja. Ya sea con un referente doméstico o de salud. En otras palabras, incluye aquellas situaciones que no son propias del rol como estudiante o empleado. Ejemplo: "Pedir dinero a mis papás" o "La situación con mi novia".

De esta manera las situaciones reportadas por los estudiantes fueron (tabla 2):

\section{Tabla 2}

Tipo de situaciones estresantes reportadas por los participantes

\begin{tabular}{lcr}
\hline & Frecuencia & $\%$ \\
\hline Académico & 14 & 46.66 \\
Personal & 3 & 10 \\
Laboral & 1 & 3.33 \\
Académico-laboral & 1 & 3.33 \\
Académico-personal & 8 & 26.66 \\
Personal-laboral & 1 & 3.33 \\
Escuela-trabajo-familia & 2 & 6.66 \\
Total & 30 & 100 \\
\hline
\end{tabular}

Inicialmente fueron sometidas a un análisis de normalidad las variables objetivo a fin de determinar la posibilidad de utilizar medidas paramétricas en los análisis posteriores, tras corroborar con la prueba Kolmogorov-Smirnov la inexistencia de diferencias significativas en contraste con una distribución normal, se procedió a realizar pruebas paramétricas en el resto de los análisis. Para conocer si los integrantes de cada grupo eran semejantes entre sí, se realizó la prueba con los datos recogidos en el pretest de las variables "estrés percibido", "emociones positivas" y "emociones negativas". Los resultados de la comparación de las características de las variables dependientes "estrés" y "emociones" en los seis grupos experimentales antes de la intervención fueron: 
$\mathrm{F}(1.5)=2.04, p=.109$ para la variable de "estrés percibido"; $\mathrm{F}(1.5)=1.98$, $p=.118$ para la variable de "emociones positivas"; $\mathrm{F}(1.5)=.82, p=.548$ para la variable de "emociones negativas". Estos resultados comprobaron la ausencia de diferencias estadísticamente significativas entre los grupos antes de la intervención.

Los efectos a un nivel general en estas mismas variables fueron analizados mediante una prueba $t$ de Student para datos pareados. Tras la intervención, no hubo una disminución significativa en el estrés percibido: $t(29)=1.35, p=.185$. Pero se encontró una disminución significativa en las emociones positivas: $t(29)=2.14, p=.04$ y también en las emociones negativas: $t(29)=4.54, p=.001$. Las medias y DE relacionadas con estas comparaciones se muestran en la tabla 3, misma que permite distinguir la existencia de disminuciones en las puntuaciones de las tres variables de estudio.

\section{Tabla 3}

Media y desviación estándar antes y después de la intervención

\begin{tabular}{|c|c|c|c|c|c|c|}
\hline & \multicolumn{2}{|c|}{ Estrés } & \multicolumn{2}{|c|}{$\begin{array}{c}\text { Emociones } \\
\text { positivas }\end{array}$} & \multicolumn{2}{|c|}{$\begin{array}{c}\text { Emociones } \\
\text { negativas }\end{array}$} \\
\hline & $M$ & $D E$ & $M$ & $D E$ & $M$ & $D E$ \\
\hline Preintervención & 24.36 & 5.79 & 33.93 & 6.80 & 21.66 & 6.87 \\
\hline Posintervención & 22.70 & 7.32 & 29.26 & 12.64 & 15.03 & 10.09 \\
\hline
\end{tabular}

Los resultados por grupo de intervención en estas mismas tres variables tras la intervención a un nivel general se muestran en la tabla 4. Como puede observarse existe una concentración en los cambios de las emociones negativas en los grupos de intervención donde se pidió a los participantes que realizaran un dibujo sobre la situación estresante seleccionada; no así en el caso de las emociones positivas, donde los cambios se encontraron a un nivel global. 


\section{Tabla 4}

Resultados de las puntuaciones de los inventarios de estrés y emociones

\begin{tabular}{lccccccc}
\hline & & \multicolumn{7}{c}{ Grupo } \\
& & 1 & 2 & 3 & 4 & 5 & 6 \\
\cline { 2 - 8 } Estrés & $M$ & 22.8 & 16.2 & 21.8 & 22.6 & 25.2 & 27.6 \\
& $D E$ & 3.96 & 4.02 & 1.78 & 10.43 & 8.1 & 9.34 \\
\multirow{2}{*}{ Emociones positivas } & $M$ & 36 & 31.8 & 22.4 & 32 & 32.6 & 20.8 \\
& $D E$ & 5.24 & 18.64 & 12.7 & 7.64 & 10.01 & 15.05 \\
Emociones negativas & $M$ & 15.8 & 12 & $\mathbf{9 . 4 0 *}$ & $\mathbf{1 6 . 0 0 *}$ & 18.8 & 18.2 \\
& $D E$ & 3.96 & 6.78 & 5.81 & 13.41 & 12.02 & 15.35 \\
\hline
\end{tabular}

${ }^{*} p \leq .05$

Nota: (1) distractor activo + narrativa; (2) distractor activo + sin narrativa; (3) enfoque + narrativa; (4) enfoque + sin narrativa; (5) distractor pasivo + narrativa; (6) distractor activo + sin narrativa.

Por otro lado la prueba multigrupo (ANOVA) mostró que en el caso del estrés percibido existía una superioridad significativa tras la intervención en el grupo que trabajó bajo la consigna de distracción pasiva sin narrativa frente al distractor activo sin narrativa, $\mathrm{F}(1.5)=2.25$, $p=.04$ En el caso de las emociones, la comparación multigrupo no evidenció superioridad en ningún tratamiento: "emociones positivas", $\mathrm{F}(1.5)=1.23, p=.32$; "emociones negativas", $\mathrm{F}(1.5)=.610$, $p=.69$

\section{Discusión}

La comparación pre post a un nivel general mostró la presencia de diferencias significativas en las emociones, tanto positivas como negativas En ambos casos se encontró una reducción. Dicho dato sugiere que, tal y como lo mencionan la APA (2010) y Linley y colaboradores (2012) actividades cotidianas como hacer un dibujo o comer un helado pueden tener un efecto positivo en las emociones. El hecho de que se haya encontrado también una reducción del afecto positivo y un cambio no significativo de estrés percibido pudiese, siguiendo estos mismos 
autores, relacionarse con que en este estudio la actividad desempeñada se vinculaba a priori a una situación estresante sobre la que habría de trabajar la persona durante el proceso experimental y no, como se recomienda en estas mismas referencias, realizadas durante el día con la finalidad de mejorar el afecto positivo. Considerando el procedimiento utilizado, otra posible causa pudiese ser que el periodo de tiempo transcurrido entre el procedimiento y la segunda evaluación solo haya dado la posibilidad de medir la activación emocional, es decir, el cambio inmediato de las emociones

Asimismo, una tercera explicación, tomando en cuenta la construcción propia del instrumento de medición para emociones utilizado y el modelo teórico que le sustenta sugeriría que el encontrarse con el aumento de ambos tipos de emociones es un dato más que de las emociones no siguen un continuum, sino que puede presentarse simultáneamente algunas pertenecientes de uno u otro tipo en un mismo tiempo o en función a un mismo evento. Asimismo, por grupo de intervención pudo observarse que en el caso de las emociones negativas existían cambios superiores en los grupos donde la intervención utilizaba el dibujo como una herramienta de enfoque al problema. Estos últimos datos corroboran los resultados de autores como Huss (2012), quien sostiene que la actividad artística otorga la oportunidad para expresar las situaciones estresantes.

Haber localizado diferencias en las distintas comparaciones grupales sobre el estrés percibido y las emociones de los participantes permite corroborar la hipótesis de que su modificación es posible mediante el empleo de una actividad artística, incluso cuando no se tiene el apoyo de un terapeuta, pero con otros referentes como es el empleo de la escritura expresiva.

Las diferencias encontradas de uno a otro análisis no solo llevan al reconocimiento del peso que la elaboración cognitiva, a manera de distinción entre instrucciones, tiene en las variables estudiadas. También proporciona algunos datos en torno al desarrollo teórico sobre el empleo de la escritura expresiva (Piolat \& Bannour, 2011), pues como se observa en la disminución encontrada con respecto al afecto negativo 
antes de realizar el dibujo sobre la situación estresante, pareciese haber pruebas ahí de la integración de la información emocional sobre las propias experiencias mencionada por estos mismos autores. Por otro lado, que el mismo resultado se obtenga sin que esta figure como parte de la intervención pudiese confirmar que fue la actividad gráfica la que promovió los cambios encontrados.

Un aspecto más que puede destacarse en relación a estos datos es que, al incorporar las actividades cotidianas para la mejora de estados afectivos (emociones o estrés) en un ambiente experimental, como sucedería en cualquier otro método de estudio, no necesariamente se confirman todos los hallazgos como tampoco se obtienen datos estadísticamente significativos en todas las variables estudiadas, lo que abre la posibilidad de indagar más en las variables asociadas que pudiesen contribuir en los datos observados empíricamente. En este caso, la dinámica encontrada desde otras teorías, por ejemplo, en el estudio de los pasatiempos como fenómeno, pudiesen contribuir ampliamente en este aspecto. El poder observar efectos distintos en cada tipo de intervención bajo la consigna de trabajar en torno a una situación personal son consistentes con el planteamiento de que las condiciones en que son utilizadas las actividades con fines de regulación emocional influyen en espacios experimentales (Webb, Miles \& Sheeran; 2012); siendo además el primer estudio en corroborarlo y ubicar diferencias en una variable vinculada: el estrés percibido.

Asimismo, contemplando la interacción entre las dos variables manipuladas (estrés y emociones) los resultados ya mencionados podrían relacionarse con la investigación de Wang y Saudino (2011), quienes seńalan que las habilidades para el afrontamiento de las situaciones estresantes y aquellas vinculadas con la regulación emocional, si bien interactúan o trabajan de manera simultánea durante una situación particular, presentan una varianza entre individuos con un correlato a explorar en función a lo heredado o lo obtenido mediante la experiencia.

Una limitante del presente estudio fue que, a pesar de la obtención de resultados significativos por grupo en todas las variables 
dependientes, no se encontró una diferencia que señalase superioridad entre unos u otros tratamientos. Esta situación abre la pauta a interrogantes sobre los factores que más allá de la manipulación experimental, permite a la persona obtener beneficios mediante estas actividades; así como la necesidad de realizar estudios más amplios que permitan distinguir, si existe, la presencia de alguna constante que permita explicar los resultados obtenidos.

En síntesis, los hallazgos del presente estudio pueden ser interpretados como evidencia favorable a la necesidad de formular protocolos estrictos cuando estos se dirigen a la investigación de intervenciones en torno a la salud, dada su influencia en el estado emocional de la persona y su percepción de las situaciones que le son relevantes. Además, dichos protocolos son promisorios en función a la incorporación de elementos teóricos provenientes de otras áreas de estudio para explicar fenómenos relacionados con la salud psicológica como son el estrés percibido y las emociones.

\section{Referencias}

American Art Therapy Association. (2013). Art therapy. Recuperado de http://www.arttherapy.org

American Psychological Association (2010). Stress in America. Washington, D.C.: American Psychological Association. Recuperado de: http://www.apa.org/news/press/releases/stress/ 2010/health-stress.pdf

Cano, F., Rodríguez, L. \& García, J. (2007). Adaptación española del inventario de estrategias de afrontamiento. Acta Española de Psiquiatria, 35(1), 29-39. Recuperado de: http://personal.us.es/ fjcano/drupal/?q=node/8

Casari, L., Anglada, J. \& Daher, C. (2014). Estrategias de afrontamiento y ansiedad ante exámenes en estudiantes universitarios. Revista de Psicología, 32(2), 243-269. 
Cantú, R. \& Álvarez, J. (2011). Expresión artistica y narrativa en pacientes con cáncer: una intervención psicológica. Nuevo León, México: Universidad Autónoma de Nuevo León.

Crawford, M. \& Patterson, S. (2007). Arts therapies for people with schizophrenia: an emerging evidence base. Evidence-Based Mental Health, 10, 69-70. http://dx.doi.org/10.1136/ebmh.10.3.69

Dalebroux, A., Goldstein, T. \& Winner, E. (2008). Short-term mood repair through art-making: Positive emotion is more effective than venting. Motivation and Emotion, 32(4), 288-295. http:// dx.doi.org/10.1007/s11031-008-9105-1

Drake, J. \& Winner, E. (2012a). Confronting sadness through artmaking: Distraction is more beneficial than venting. Psychology of Aesthetics, Creativity and the Arts, 6(3), 255-261. http://dx.doi. org/10.1037/a0026909

Drake, J. \& Winner, E. (2012b). How children use drawing to regulate their emotions. Cognition \& Emotion, 27(3), 1-9. http:// dx.doi/org/10.1080/02699931.2012.720567

Eschleman, K., Alarcon, G., Lyons, J., Strokes, C. \& Schneider, T. (2011). The dynamic nature of the stress appraisal process and the infusión of affect. Anxiety, Stress and Coping, 6(16), 1-9. http://dx.doi/org/10.1080/10615806.2011.601299

García-Ros, R., Pérez-Blasco, F. \& Natividad, L. (2012). Evaluación del estrés académico en estudiantes de nueva incorporación a la universidad. Revista Latinoamericana de Psicología, 44(2), 143154.

González-Cabrera, J., Fernández-Prada, M., Guillén-Solvas, J., MolinaRuano, R., Blazquez, A. y Peinado, J. M. (2012). Psychosocial risk at work, self-perceived stress, and salivary cortisol level in a sample of emergency physicians in Granada. Emergencias, 24, 101-106.

González, M. T. \& Landero, R. (2007). Factor Structure of the Perceived Stress Scale (PSS) in a Sample from Mexico. The Spanish Journal of Psychology, 10(1), 199-206. http://dx.doi. org/10.1017/S1138741600006466 
Huss, E. (2012). Integrating strengths and stressors through combining dynamic phenomenological and social perspectives into art evaluations. The Arts in Psychoterapy, 39, 451-455. http://dx.doi/ org/ 10.1016/j.aip.2012.07.001

Kandel, E. (2012). The Age of Insight. Nueva York: Random House.

Kerlinger, F. \& Lee, H. (2002). Investigación del comportamiento. Métodos de investigación en ciencias sociales. México: McGrawHill.

Lang, P.J., Bradley, M.M. \& Cuthbert, B.N. (2008). International affective picture system (IAPS): Affective ratings of pictures and instruction manual. Technical Report A-8. University of Florida, Gainesville, FL.

Leka, S., Griffiths, A. \& Cox, T. (2004). Work organization \& Stress. Systematic problem approaches for employers, managers and trade union representatives. Nottingham: World Health Organization.

Linley, P. A., Dovey, H., de Bruin, E., Transler, C., Wilkinson, J., Maltby, J. \& Hurling, R. (2012). Two simple, brief, naturalistic activities and their impact on positive affect: feeling grateful and eating ice cream. Psychology of Well-Being: Theory, Research and Practice, 3(6), 1-14. http://dx.doi.org/10.1186/2211-1522-3-6

Martín, I. (2007). Estrés académico en estudiantes universitarios. Apuntes de Psicología, 25(1), 87-99.

Marxen, E. (2011). Diálogos entre arte y terapia. Del "arte psicótico" al desarollo de la arteterapia y sus aplicaciones. Barcelona, España: Gedisa.

Montes, C., Rodríguez, D. \& Serrano, G. (2014). Estrategias de manejo de conflicto en clave emocional. Anales de Psicología, 39(1), 238246. http://dx.doi.org/10.6018/analesps.30.1.135171

Muri, S. (2007). Beyond the face: Art therapy and self-portraiture. The Arts in Psychotherapy, 34, 331-339. http://dx.doi.org/10.1016/j. aip.2007.05.002

Piolat, A. \& Bannour, R. (2011). Les effets de l'écriture expressive sur la santé physique et psychologique des rédacteurs: un bilan, des perspectives de recherches. Revuew européenne de psycologie appliquée, 61, 101-113. doi: 10.1016/j.erap.2010.12.003 
Reynolds, F. (2010). 'Colour and communion': Exploring the influences of visual art-making as a leisure activity on older women's subjective well-being. Journal of Aging Studies, 24, 135-143. http://dx.doi.org/10.1016/j.jaging.2008.10.004

Robles, R. \& Páez, F. (2003). Estudio sobre la traducción al español y las propiedades psicométricas de las escalas de afecto positivo y negativo (PANAS). Salud Mental, 26(1), 69-75.

Szczygiel, D., Buczny, J. \& Bazinka, R. (2012). Emotion regulation and emotional information processing: The moderating effect of emotional awareness. Personality and Individual Differences, 52(3), 433-437. http://dx.doi.org/10.1016/j.paid.2011.11.005

Singer, S., Götze, H., Buttstädt, M., Geue, K., Momenghalibaf, A. \& Böhler, U. (2010). The effects of an art education program on competencies, coping, and well-being in outpatients with cancer: Results of a prospective feasibility study. The Arts in Psychotherapy, 37, 363-369. http://dx.doi.org/10.1016/j. aip.2010.07.002

Wang, M. \& Saudino, K. (2011). Emotion Regulation and Stress. Journal of Adult Development, 18, 95-103. http://dx.doi. org/10.1007/S10804-010-91147

Webb, T., Miles, E. \& Sheeran, P. (2012). Dealing with feeling: A meta-analysis of the effectiveness of strategies derived from the process model of emotion regulation. Psychological Bulletin, 138(4), 775-808. http://dx.doi.org/10.1037/a0027600

Recibido el 13 de mayo, 2015 Aceptado el 12 de abril, 2016 